\title{
Coherence and scale of vertical velocity in the convective boundary layer from a Doppler lidar
}

\author{
Marie Lothon • Donald H. Lenschow • \\ Shane D. Mayor
}

\begin{abstract}
We utilized a Doppler lidar to measure integral scale and coherence of vertical velocity $w$ in the daytime convective boundary layer (CBL). The high resolution $2 \mu \mathrm{m}$ wavelength Doppler lidar developed by the NOAA Environmental Technology Laboratory was used to detect the mean radial velocity of aerosol particles. It operated continuously in the zenith-pointing mode for several days in the summer 1996 during the "Lidars in Flat Terrain" experiment over level farmland in central Illinois. We calculated profiles of $w$ integral scales in both the alongwind and vertical directions from about $390 \mathrm{~m}$ height to the CBL top. In the middle of the mixed layer we found, from the ratio of the $w$ integral scale in the vertical to that in the horizontal direction, that the $w$ eddies are squashed by a factor of about 0.65 as compared to what would be the case for isotropic turbulence. Furthermore, there is a significant decrease of the vertical integral scale with height. The integral scale profiles and vertical coherence show that vertical velocity fluctuations in the CBL have a predictable anisotropic structure. We found no significant tilt of the thermal structures with height in the middle part of the CBL.
\end{abstract}

Keywords Coherence - Convective boundary layer · Integral scale · Lidar . Turbulence

The National Center for Atmospheric Research is sponsored by the National Science Foundation.

M. Lothon $(\varangle) \cdot$ D. H. Lenschow · S. D. Mayor

National Center for Atmospheric Research, Boulder,

Colorado, USA

Present address: M. Lothon

Laboratoire d'Aérologie, Toulouse, CNRS,

Centre de Recherches Atmosphériques,

8 route de Lannemezan, 65300

Campistrous, France

e-mail: lotm@aero.obs-mip.fr 


\section{Introduction}

Turbulence is the main transport mechanism occurring within the convective boundary layer $(\mathrm{CBL})$. Surface-layer processes, driven mostly by solar heating and wind shear, drive the exchange of latent heat, sensible heat and mass between the surface and the free troposphere. This exchange occurs over a broad spectrum of scales, but the largest contributions are due to eddies that scale with the CBL depth.

One way to investigate the structure of turbulence from observational studies is to consider the distance over which, on average, a variable remains correlated with itself. Published estimates of this scale were obtained from airplane in situ measurements (e.g., Lenschow and Stankov, 1986; Durand et al., 2000) because of the ability of aircraft to probe from a few decametres above the surface to the free troposphere, and cover long distances in a relative short period of time. A major limitation, however, is that an aircraft can only probe one level at a time, and thus cannot measure two-point turbulence statistics on scales larger than the transverse dimensions of the aircraft.

An exception to this is the work of Lenschow and Kristensen (1988) and Kristensen et al. (1989), who flew two identical aircraft in formation during the Dual Aircraft Formation Flight Experiment (DAFFEX) to obtain lateral two-point velocity statistics of all three wind components in the CBL. They also flew the two aircraft vertically displaced to measure vertical two-point velocity statistics (Davis, 1992). Similarly, Kristensen et al. (1989) used measurements from three towers arrayed roughly normal to the wind during the Lammefjord Experiment (LAMEX) to obtain two-point statistics in the atmospheric surface layer.

Mann (1994) presents a detailed discussion of second-order turbulence structure in the neutral atmospheric surface layer and develops a model of two-point statistics that uses the isotropic turbulence spectrum of von Kármán. Applications of two-point statistics include estimating fluctuating loads on structures due to spatial variations in the turbulent velocity components, and calculating sampling requirements in order to estimate error variances in spatially averaged wind field variables such as divergence and vorticity (e.g., Lenschow et al., 1999).

With the development of instruments for remotely sensing velocity, such as Doppler radars and lidars, it is now possible to measure the radial velocity component as a function of distance from the transmitter and thus to map out two-dimensional fields of radial velocity. Here we report on measurements of vertical velocity $w$ statistics from a ground-based zenith-pointing Doppler lidar deployed over a relatively flat and uniform agricultural surface. Vertical cross-sections of $w$ are used to calculate the integral scale and vertical coherence at various separation distances for 11 daytime CBL cases. As far as we know, this is the first time that such a study has been carried out. We compare our results with the predicted coherence for inertial subrange turbulence and for a von Kármán isotropic turbulence spectrum.

\section{Experiment and instrumentation}

\subsection{Lidars-in-Flat-Terrain experiment}

During August 1996, the National Center for Atmospheric Research's Atmospheric Technology Division (NCAR/ATD) and NOAA's Environment Technology Laboratory (ETL) deployed three lidars at the University of Illinois field site near Champaign, 
Illinois, USA, to observe the high resolution structure of aerosol, winds, and ozone in the lowest few kilometres of the atmosphere as the CBL evolved from early morning to late evening. The site for Lidars-In-Flat-Terrain (LIFT) was chosen because of the flat terrain, good aerosol scattering, and nearby UHF radar wind profilers operated by the NOAA Aeronomy Laboratory. In addition to the lidars and permanent wind profilers, surface-based meteorological instrumentation and additional wind profilers were deployed, and radiosondes were launched on a regular basis (Cohn et al., 1998). Angevine et al. (1998) have summarized the concurrent Flatland Boundary Layer experiment, which shared instruments and had complementary objectives.

\subsection{High resolution Doppler lidar}

One of the three lidars, the High Resolution Doppler Lidar (HRDL), was used for this study. It was developed and deployed by ETL and is described by Grund et al. (2001), and utilizes a solid-state thulium lutetium yttrium aluminium garnet (Tm:Yu, YAG) laser to generate coherent infrared pulses at $2.0218 \mu \mathrm{m}$ wavelength that are transmitted and received by a $0.2 \mathrm{~m}$ telescope at a pulse repetition rate of $200 \mathrm{~s}^{-1}$. A beam-steering mechanism installed on the roof of the shipping container housing the lidar allowed pointing and scanning anywhere above the horizon. During LIFT, the laser generated $0.8 \mathrm{~mJ}$ pulses with a radial resolution of $30 \mathrm{~m}$, and a minimum range (dead-zone) of about $390 \mathrm{~m}$. Typically, the lidar was able to "see" several kilometres horizontally and, at the zenith, was always able to see through the top of the CBL. Changes in aerosol scattering led us to vary the number of pulses averaged together, and thus the temporal resolution (from one to few seconds) on a daily basis.

Although the HRDL was used in various scanning modes during LIFT, a majority of the observations (110 out of over 160 hours) were with the laser beam pointing straight up, since a major focus of LIFT was to examine the vertical structure of $w$ in a CBL. This takes advantage of the lidar's capability to obtain range-resolved radial measurements, from which a two-dimensional field of $w$ can be obtained by use of Taylor's hypothesis; that is by assuming that the field of turbulence is "frozen" as it advects past the lidar.

\subsection{Description of selected cases}

Here we show results from 11 cases with useful vertical HRDL data collected during LIFT and differing CBL scaling variables (mean wind, CBL depth, and stability). Table 1 summarizes characteristics for each case averaged over the selected period of time (approximately centred in the middle of the day) that was chosen for the analysis of integral scales and coherence. The periods were selected on the basis of data continuity and quality, and stationarity of the CBL. On most of the days, fair-weather $\mathrm{Cu}$ formed by late morning. Profiles of the horizontal mean wind $U$ were obtained from the wind profiler located at Sadorus, IL, about $5 \mathrm{~km}$ from the HRDL; in Table 1 they have been averaged over the entire CBL for the selected period. Figure 1 shows time-height cross-sections of $w$ measured by the HRDL during two different days with different wind conditions. Horizontal wind profiles measured by the profiler are also shown. On 16 August, the wind was weak, and within the three hours of observations, only a few thermals passed through the beam; but the time resolution $\left(1 \mathrm{~s}^{-1}\right)$ and data quality are high. On 19 August, the wind was stronger and improved the sampling 
Table 1 Characteristics of the 11 LIFT cases considered here. $z_{i}$ is the CBL depth, $U$ is the mean horizontal wind, $L_{O}$ is the Obukhov length, $l_{w}^{(x)}$ is the horizontal integral scale at $z_{i} / 2$, and $l_{w}^{(z)}$ is the vertical integral scale at $z_{i} / 2.1700 \mathrm{UTC}$ is $1100 \mathrm{CST}$

\begin{tabular}{lllllllll}
\hline $\begin{array}{l}\text { Date } \\
(\text { mmddyy })\end{array}$ & $\begin{array}{l}\text { time period } \\
(\mathrm{UTC})\end{array}$ & $z_{i}(\mathrm{~m})$ & $U\left(\mathrm{~m} \mathrm{~s}^{-1}\right)$ & $-z_{i} / L_{o}$ & $\begin{array}{l}l_{w}^{(x)\left(z_{i} / 2\right)} \\
(\mathrm{m})\end{array}$ & $\begin{array}{l}l_{w}^{(x)\left(z_{i} / 2\right)} \\
(\mathrm{m})\end{array}$ & $l_{w}^{(x)} / z_{i}$ & $l_{w}^{(x)} / z_{i}$ \\
\hline 080296 & $1700-2000$ & 1590 & 3.0 & 91 & 230 & 372 & 0.14 & 0.23 \\
080496 & $1700-2000$ & 1440 & 5.2 & 26 & 198 & 287 & 0.14 & 0.20 \\
080596 & $1700-2000$ & 1190 & 8.6 & 6 & 154 & 222 & 0.13 & 0.19 \\
080696 & $1800-2100$ & 1390 & 7.8 & 15 & 462 & 552 & 0.33 & 0.40 \\
080796 & $1800-2100$ & 1270 & 5.6 & 13 & 320 & 419 & 0.25 & 0.33 \\
081096 & $1700-2100$ & 1770 & 2.2 & 121 & 311 & 421 & 0.18 & 0.24 \\
081296 & $1800-2100$ & 1720 & 4.8 & 23 & 497 & 609 & 0.29 & 0.35 \\
081696 & $1800-2100$ & 1370 & 2.2 & 251 & 398 & 541 & 0.29 & 0.39 \\
081996 & $1800-2000$ & 1280 & 7.2 & 11 & 312 & 419 & 0.24 & 0.33 \\
082096 & $1800-2100$ & 960 & 6.8 & 14 & 175 & 233 & 0.18 & 0.24 \\
082196 & $1800-2100$ & 1530 & 3.4 & 70 & 293 & 353 & 0.13 & 0.19 \\
\hline
\end{tabular}
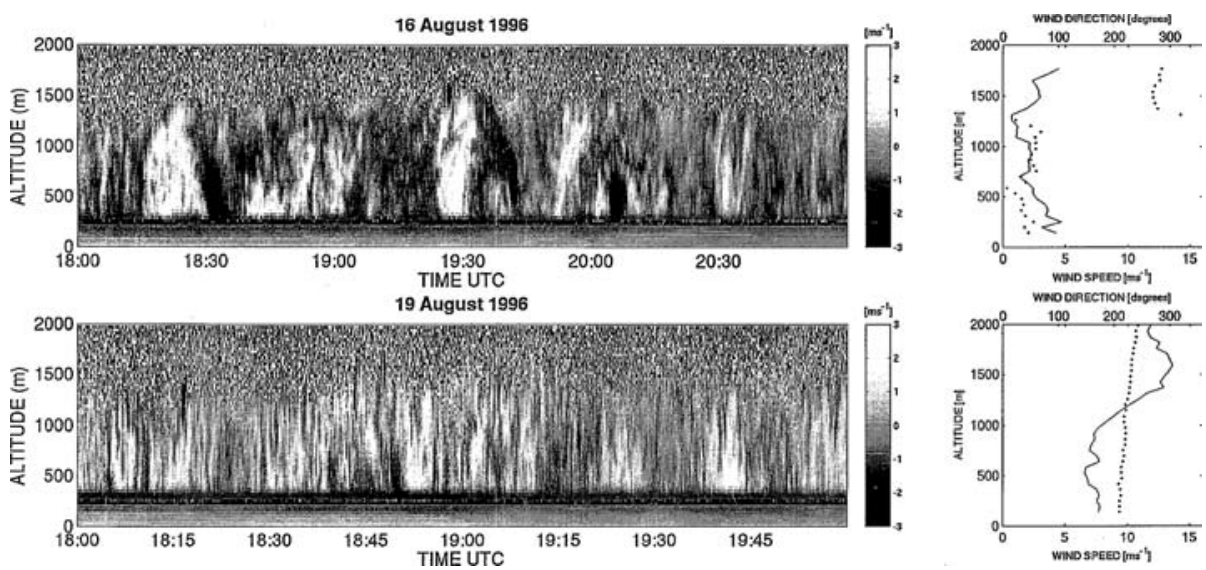

Fig. 1 Left panels: Height-time cross-section of $w$ measured by the HRDL on August 16 (top) and 19 (bottom). Right panel: mean profile of the horizontal wind speed (solid line) and direction (dotted line) measured by the UHF profiler located nearby, and averaged over the time periods shown in the left panel

statistics of our analysis. For most of the cases there is little evidence for a systematic wind shear across the depth of the CBL.

The CBL top $z_{i}$ was determined from the height at which the increase in variance over 1-minute segments first exceeded $0.7 \mathrm{~m}^{2} \mathrm{~s}^{-2}$ over a height increment of $30 \mathrm{~m}$. That is, when the aerosol backscatter first becomes too weak to provide a measurable velocity and the signal is dominated by noise.Thus, we assume that $z_{i}$ is a demarcation between a particulate-laden CBL and a relatively clean free atmosphere. This criterion also identifies cloud base when fair-weather $\mathrm{Cu}$ are growing out of the CBL top. The values of $z_{i}$ in Table 1 are obtained from an average over the given period. These estimates of $z_{i}$ compare well with independent estimates from the nearby wind profilers and with the analyses of Cohn and Angevine (2000), and Grimsdell and Angevine (1998, 2002). 


\section{Lateral coherence of a stationary homogeneous flow}

Coherence is a useful tool for documenting spatial correlation between random stationary time series. Following the definition by Kristensen and Jensen (1979, hereafter $\mathrm{KJ}$ ), the coherence of a velocity component $u_{i}$ separated by a vector $\mathbf{D}$ from a velocity component $u_{j}$ is

$$
\operatorname{Coh}_{i j}(\mathbf{D}, k) \equiv \frac{\operatorname{Co}_{i j}(\mathbf{D}, k)^{2}+Q_{i j}(\mathbf{D}, k)^{2}}{F_{i i}(k) F_{j j}(k)},
$$

where $k$ is the wavenumber, $F_{i i}(k)$ and $F_{j j}(k)$ are the spectra of the individual velocity component time series, and $C_{i j}(\mathbf{D}, k)$ and $Q_{i j}(\mathbf{D}, k)$ are the cospectra and quadrature spectra. Additional information is provided by the phase angle, defined by

$$
\phi_{i j}(\mathbf{D}, k) \equiv \arctan \left(\frac{Q_{i j}(\mathbf{D}, k)}{C o_{i j}(\mathbf{D}, k)}\right) .
$$

Here, the mean wind direction defines the direction of the first unit vector $\mathbf{i}_{\mathbf{1}}$, and the displacement $D$ along the vertical defines the second unit vector $\mathbf{i}_{2}$. We note that $0 \leq \operatorname{Coh}_{i j}(\mathbf{D}, k) \leq 1$.

For large $k$ and $\mathbf{D}$, the coherence becomes small, as the eddies become independent of each other. In contrast, for small $k$ and $\mathbf{D}$ the coherence approaches one. When dealing with finite measurement periods, there is a statistical uncertainty in estimating coherence. Since $\operatorname{Coh}_{i j}(\mathbf{D}, k) \geq 0$, this uncertainty results in a positive bias, which increases as the number of Fourier modes that are averaged together and the length of the time series decreases. For one Fourier mode, the coherence is identically one. However a time series can be subdivided into a set of $M$ time series and the coherence calculated over a set of Fourier modes. The product of these two numbers is often considered as the number of degrees-of-freedom $d f$ for estimating the significance of the measured coherence. Kristensen and Kirkegaard (1986) addressed the issue of how large $d f$ should be for a given level of significance in coherence estimates.

We first consider the coherence of the vertical velocity along the vertical axis $z$ obtained from the HRDL data at two different heights (i.e., two different range gates) within the CBL. Using the notation of KJ, the coherence that we can estimate from these measurements is $\operatorname{Coh}_{22}(D, k)$, where $D$ is the vertical separation distance. If the turbulence is homogeneous and isotropic, $\mathrm{KJ}$ show how to obtain $\operatorname{Coh}_{i j}(D, k)$ analytically from the energy spectrum $E(k)$. If we further assume that $D$ is much smaller than the scale of the turbulence $L$, the coherence is close to one for $D k \ll 1$, independent of the behaviour of the spectrum at wavenumbers $k \ll 1 / L$, so that the energy spectrum can be approximated by the Kolmogorov spectrum

$$
E_{k}(k)=\alpha \varepsilon^{2 / 3} k^{-5 / 3},
$$

where $\alpha$ is the Kolmogorov constant and $\varepsilon$ is the rate of dissipation of turbulent kinetic energy. In this case, $\operatorname{Coh}_{22}(D, k)$ can be obtained analytically and is a function of the normalized variable $D k(\mathrm{KJ})$ :

$$
\begin{gathered}
\operatorname{Coh}_{22}(\mathrm{D}, k)=\operatorname{Coh}_{22}(\mathrm{D} k)=(\Gamma(5 / 6))^{-2}\left(\frac{D k}{2}\right)^{5 / 3} \\
\times\left(2 K_{5 / 6}(D k)+\frac{3}{4} D k K_{1 / 6}(D k)\right)^{2},
\end{gathered}
$$


where $K$ is the modified Bessel function of the second kind (Luke, 1972) and $\Gamma$ is the Gamma function. This theoretical Kolmogorov coherence spectrum is shown in Figs. 9 and 10.

When $D$ can no longer be assumed small compared to $L$, the expression for the coherence depends not only on $D k$ but also on $D / L$. Assuming a von Kármán energy spectrum with a length scale $L$

$$
E_{v}(k)=\alpha \varepsilon^{2 / 3} \frac{L^{17 / 3} k^{4}}{\left(1+k^{2} L^{2}\right)^{17 / 6}},
$$

we obtain an analytical expression for $\operatorname{Coh}_{22}(D / L, D k)$,

$$
\begin{gathered}
\operatorname{Coh}_{22}(D / L, D k)=9 \frac{2^{1 / 3} S^{11 / 3}}{(\Gamma(5 / 6))^{2}\left(3 \frac{D^{2}}{L^{2}}+8 D^{2} k^{2}\right)^{2}} \\
\times\left(\left(D^{2} k^{2} K_{11 / 6}(S)+S K_{5 / 6}(S)\right)^{2},\right.
\end{gathered}
$$

where $\mathrm{S}^{2}=\frac{D_{2}}{L^{2}}+D^{2} k^{2}$.

Figure 2 shows how $\operatorname{Coh}_{22}(D / L, D k)$ monotonically decreases as $D k$ and $D / L$ increase. One important aspect of the coherence is that for significant displacement $D$ relative to $L$, the coherence is not equal to one for $D k=0$. It can easily be shown that (6) becomes identical to (4) in the limit $D / L \rightarrow 0$.

\section{Effect of beam averaging}

Lidar velocity measurement is not a point measurement of the velocity field but an average over the resolution volume that depends on the pulse width, range-gate length and time resolution. The beam can be approximated by an infinitely narrow cylinder so that the effect of the beam averaging on the $w$ measurement can be analytically studied assuming Taylor's hypothesis and Kolmogorov or von Kármán isotropic turbulence models (Frehlich, 1997; Frehlich et al., 2006).

Fig. 2 Lateral coherence $\mathrm{Coh}_{22}(D / L, D k)$ contours assuming a von Kármán energy spectrum

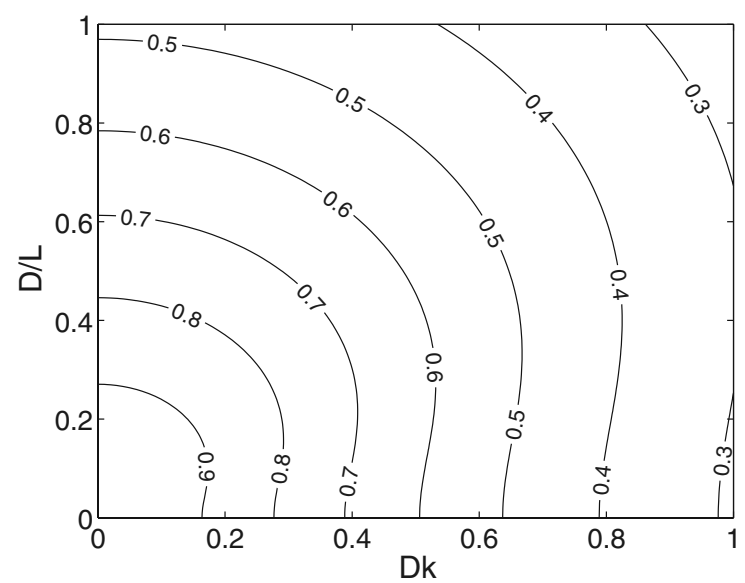


We use the model developed by Frehlich et al. (2006) to evaluate the effect of beam averaging on our estimates of integral scales. The lidar characteristics assumed here (Michael Hardesty, personal communication, 2005) are a 30-m pulse width and $30-\mathrm{m}$ range-gate length. We computed the theoretical autocorrelation functions using a 30-m lag for the covariance along the vertical, and 5-m lag along the horizontal (assuming a $5 \mathrm{~m} \mathrm{~s}^{-1}$ mean horizontal wind and $1 \mathrm{~s}^{-1}$ sampling rate). Frehlich et al. (2006) derive the theoretical autocorrelation function assuming a von Kármán energy spectrum parameterized by the variance and integral scale of $w$ and taking account of the beam averaging. We use a $1 \mathrm{~m}^{2} \mathrm{~s}^{-2}$ variance and the observed integral scales. Of course, the measured parameters are already affected by beam averaging, so we then calculated a second estimate of the integral scale from this theoretical autocorrelation function using the same exponential-fit method as was used with the measurements (see Sect. 5). The theoretically corrected estimates of the $w$ integral scale in the vertical $l_{w}^{(z)}(z)$ and the $w$ integral scale in the alongwind direction $l_{w}^{(x)}(z)$ (defined in Sect. 5) are $\simeq 10 \%$ and $\simeq 15 \%$ greater, respectively, than the measured values.

Of course, this analysis is only approximate since the von Kármán model assumes isotropic turbulence and the measurements discussed later show that the $w$ turbulence is anisotropic. But since the beam averaging effect is not large, and is similar in magnitude for both $l_{w}^{(z)}(z)$ and $l_{w}^{(x)}(z)$, we consider beam averaging to have a minor effect on the integral scale measurements and a negligible effect on their ratios.

Taking account of the beam averaging for the coherence is more problematical since we have no simple analytical approach to take account of it. However, we did use some higher resolution aircraft $w$ measurements to empirically study the effect of beam averaging on coherence. Only the denominator in (1) is sensitive to this effect, and the effect on spectra is only significant for larger $k$ than what we considered here, so we conclude that its effect on our coherence measurements is negligible.

\section{Integral scales}

To calculate the integral scales, we make use of the autocorrelation function $R_{w}(r)$. The integral scale of $w$, which is a measure of the length over which $w$ is relatively well correlated with itself, is defined as:

$$
l_{w}=\int_{0}^{\infty} \frac{R_{w}(r)}{R_{w}(0)} d r,
$$

where $r$ the displacement and

$$
R_{w}(r) \equiv \int_{-\infty}^{\infty} w\left(r^{\prime}\right) w\left(r^{\prime}+r\right) d r^{\prime}
$$

A good estimate of $l_{w}$ can be obtained from the maximum of the running integral of (7) (Lenschow and Stankov, 1986):

$$
l_{w}(r) \cong\left[\int_{0}^{r} \frac{R_{w}\left(r^{\prime}\right)}{R_{w}(0)} d r^{\prime}\right]_{\max },
$$

which is reached at the first zero-crossing of $R_{w}(r)$. Kristensen et al. (1989) show that for the isotropic von Kármán turbulence spectrum the length scale $L$ in (5) is 
proportional to the integral scales; i.e., for the transverse integral scale $l_{w}^{(x)}(z)$ :

$$
L=\left(\frac{2}{\pi}\right) \frac{\Gamma(1 / 2) \Gamma(1 / 3)}{\Gamma(5 / 6)} l_{w}^{(x)}(z) \simeq 2.68 l_{w}^{(x)}(z) .
$$

and for the longitudinal integral scale $l_{w}^{(z)}(z)$,

$$
L=\frac{\Gamma(1 / 2) \Gamma(1 / 3)}{\pi \Gamma(5 / 6)} l_{w}^{(z)}(z) \simeq 1.34 l_{w}^{(z)}(z) .
$$

Integral scales of $w$ can be estimated along both the alongwind and vertical directions:

- The alongwind integral scale $l_{w}^{(x)}(z)$ at level $z$ is calculated from $R_{w}(z, x)$, where $x=U t$.

- The vertical integral scale $l_{w}^{(z)}(z)$ is obtained from $R_{w}(z, \delta z)$. In this case, we consider a reference level $z$, and calculate the correlation coefficient as a function of the height $z$ and $\delta z$, where $\delta z$ is the varying height increment above the level $z$, between the time series at $z$ and the time series of the levels above. Shifting the level $z$ provides a profile of $l_{w}^{(z)}(z)$. Note that in this case, the height $z$ is the lower limit of the height interval over which $l_{w}^{(z)}(z)$ is calculated.

Previously Lenschow and Stankov (1986) estimated $l_{w}^{(x)}$ from the first zero-crossing of $R_{w}(z, x)$ and (9). But this method could not be consistently used to estimate $l_{w}^{(z)}(z)$ because of the limited range of values of $\delta z$ in $R_{w}(z, \delta z)$, which are restricted to about $\delta z \leq z-0.7 z_{i}$ due to temporal and spatial changes in $z_{i}$, and noise, with the added lidar dead-zone limitation of $z>390 \mathrm{~m}$. So instead, we use an exponential least squares fit,

$$
R_{w}(z, \delta z)=R_{w}(z, 0) \exp ^{-\delta z / l_{w}}
$$

to estimate $l_{w}^{(z)}(z)$. To justify this, we compare the estimates of $l_{w}^{(x)}(z)$ using both techniques since the time series are always long enough for $R_{w}(z, x)$ to cross zero (see Fig. 4). Recognizing that the raw lidar data contain random uncorrelated noise (Lenschow et al., 2000), before estimating $l_{w}$ the noise contribution was estimated and removed by extrapolating $R_{w}$ to zero lag. Then the exponential fit was made on the corrected renormalized $R_{w}$ over the lags for which $R_{w}>0.5$ in order to obtain an objective estimate of $l_{w}$.

Figure 3 shows examples of $R_{w}(x)$ at $z_{i} / 2$ and $R_{w}\left(z_{1}\right), z_{1}>0$, where $z_{1}=z-0.4 z_{i}$, for the 3-hour period on 21 August 1996 indicated in Table 1. Both are well fitted by an exponential for separation distances $<1 \mathrm{~km}$. Of course, they cannot be perfect fits, since an exponential autocorrelation function implies a $k^{-2}$ spectrum.

We then compare both the classical first zero-crossing and the exponential fit methods to obtain $l_{w}^{(x)}(z)$. For most cases, both methods give the same estimates (see Fig. 4). For two cases (19 and 6 August), the exponential method gives significantly smaller integral scales because, for large $x, R_{w}(z, x)$ decreases to zero more slowly than the extrapolated exponential fit at small $x$. Inspection of the time series indicates that this is the result of larger-scale coherent structures in the velocity field, which inevitably leads to some arbitrariness in characterizing the integral scale.

Both $l_{w}^{(x)}\left(z_{i} / 2\right)$ and $l_{w}{ }^{(z)}\left(z_{i} / 2\right)$ are shown in Table 1 . Figure 5 shows $1_{w}^{(z)} / z_{i}$ versus $l_{w}{ }^{(x)} / z_{i}$ for all days and for levels contained within a $250-\mathrm{m}$ thick layer centred at $z_{i} / 2$. The two scales are very well correlated and the ratio is remarkably constant 

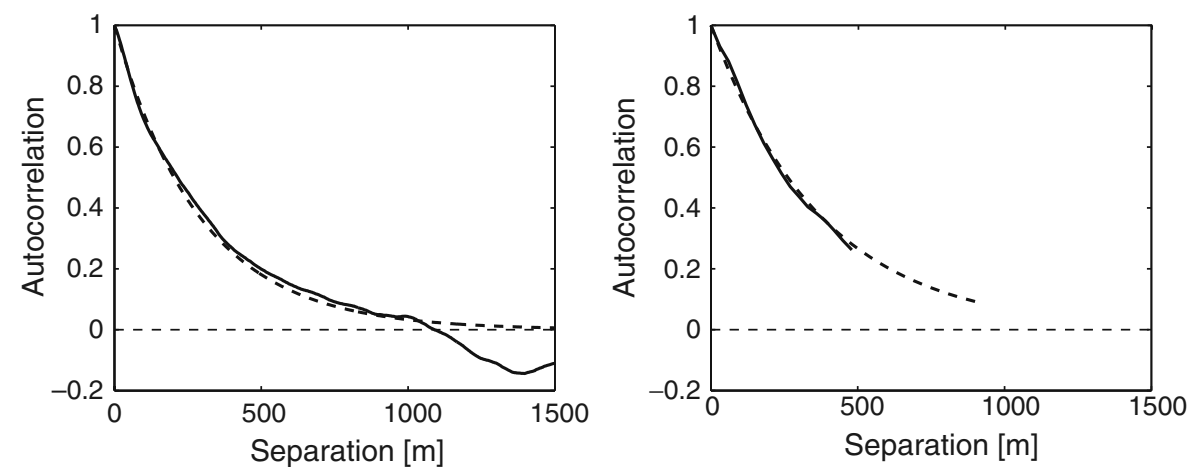

Fig. 3 Top panel: $R_{w}\left(z_{i} / 2, x\right)$ for a 3-hour time series on 21 August 1996. Bottom panel: $R_{w}\left(z_{1}\right), z_{1}>$ 0 , where $z_{1}=z-0.4 z_{i}$ for a 3-hour time series on 21 August 1996. The dashed lines are exponential functions that best fit the observations

Fig. 4 Comparison of $l_{w}^{(x)}(z)$ estimated with the first zero-crossing method and with the exponential least squares fit. Only levels in the middle of the CBL are considered, i.e., four levels below and four levels above $z_{i} / 2$, a height interval of $250 \mathrm{~m}$

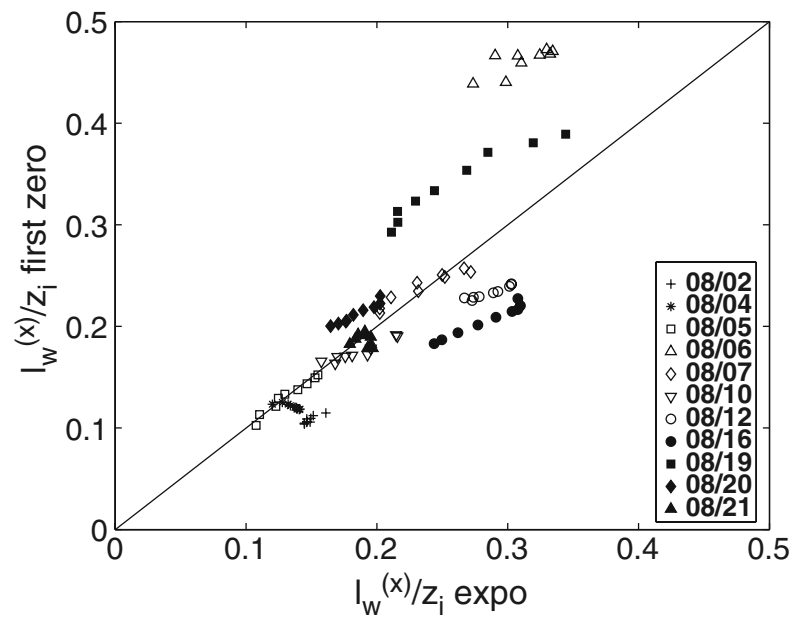

over all the cases. According to predictions of isotropic turbulence, $l_{w}^{(z)}$ should be twice as large as $l_{w}^{(x)}$ (Batchelor, 1953). We find instead that $l_{w}^{(z)}(z) \simeq 1.3 l_{w}^{(x)}(z)$; that is, $l_{w}^{(z)}(z) / l_{w}^{(x)}(z)$ is 0.65 times what it would be for isotropic turbulence. Thus, the $w$ eddies are "squashed" in the vertical direction even in the middle of the mixed layer and the amount of squashing is independent of $z_{i} / L_{o}$, where $L_{o}$ is the Obukhov length.

Figures 6 and 7 display the vertical profiles of, respectively, $l_{w}^{(x)}(z) / z_{i}$ and $l_{w}^{(z)}(z) / z_{i}$ for the 11 days. The empirically estimated profile of $l_{w}^{(x)}(z) / z_{i}$ found by Lenschow and Stankov (1986) from aircraft observations during the Air Mass Transformation Experiment (AMTEX), $l_{w}^{(x)}(z) / z_{i}=0.24\left(z / z_{i}\right)^{1 / 2}$, is also plotted. The profiles that we observed are of similar magnitude to those observed in AMTEX in the middle of the CBL, but are larger than the AMTEX values below that. In contrast to the increase with height obtained in AMTEX, we see here a nearly constant value of $l_{w}^{(x)}(z)$ throughout the height range observed here. We speculate that this may be due to the heterogeneity of the surface (a patchwork of soybean and corn fields) in LIFT 
Fig. $5 l_{w}^{(z)}(z)$ versus $l_{w}^{(x)}(z)$ for the 11 LIFT cases. Only levels in the middle of the CBL are considered; that is, four levels below and four levels above $z_{i} / 2$, as in Fig. 4. The solid line is the 2/1 slope characteristic of isotropic turbulence. The dashed line is a linear least-squares fit to the data, constrained to intercept zero

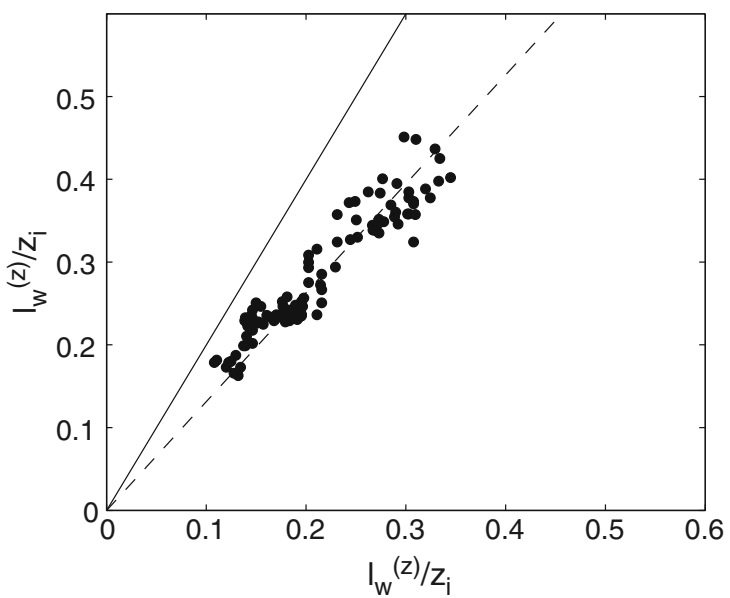

Fig. 6 Profiles of $l_{w}^{(x)}(z)$ for $0.2<z / z_{i}<0.8$ for the 11 LIFT cases. A composite $R_{W}(z, x)$ obtained from the average of $R_{w}(z, x)$ at five successive levels was calculated before calculating $l_{w}^{(x)}(z)$. The dashed line is the empirical fit obtained by Lenschow and Stankov (1986)

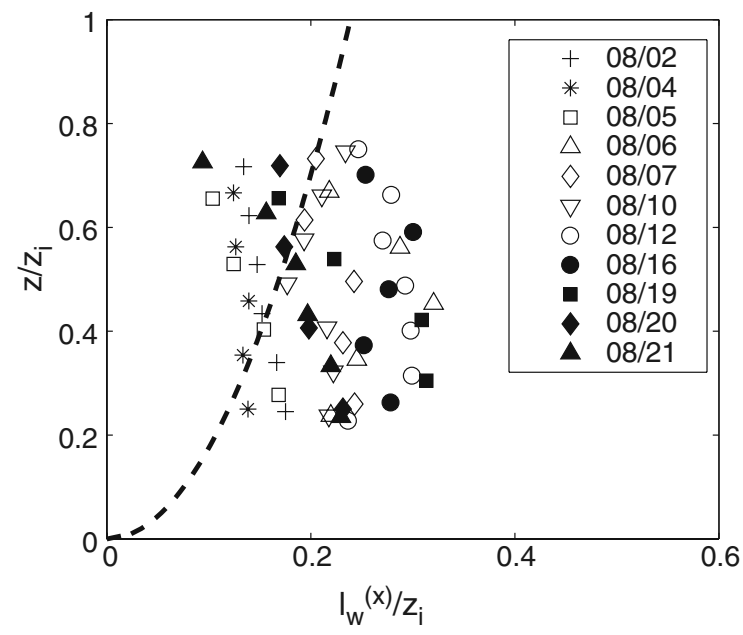

that may generate larger-scale fluctuations near the surface. We also suggest that the presence of some stratiform clouds in AMTEX may have generated larger-scale fluctuations near the CBL top.

Angevine et al. (1998) show the surface virtual temperature flux at noon each day for the entire summer for both a corn and a soybean field. They found the temperature flux over the soybean field to be as much as a factor of two higher than over the corn field till mid July, when this systematic difference virtually disappeared. They concluded that the temperature flux differences were mostly related to the maturity of the crops. This result is similar to that of Prueger et al. (2003) who found up to a factor of two difference between latent and sensible heat fluxes over corn versus soybean fields in Iowa, USA, for several days in July.

In contrast to $l_{w}^{(x)}(z)$, for $z / z_{i}>0.3$ we see a significant decrease of $l_{w}^{(z)}(z)$ with height (Fig. 7). Here we have no previous observational data with which we can compare. We note, however, that this is consistent with the eddies being squashed as they approach the top of the CBL (Kristensen et al., 1989). 
Fig. 7 Profiles of $l_{w}^{(z)}(z)$ measured from a reference level (in ordinate) just above the dead zone $(\simeq 390 \mathrm{~m})$ for the 11 LIFT cases. Every fifth level is plotted
Fig. 8 Profiles of $l_{w}^{(z)}\left(z / z_{i}\right) / z_{i}$ (triangles) and $l_{w}^{(x)}\left(z / z_{i}\right) / z_{i}$ (squares) averaged over the 11 LIFT cases. The dashed line is the empirical fit obtained by Lenschow and Stankov (1986) for $l_{w}^{(x)}\left(z / z_{i}\right) / z_{i}$. The horizontal lines represent the standard deviation over the set of considered cases
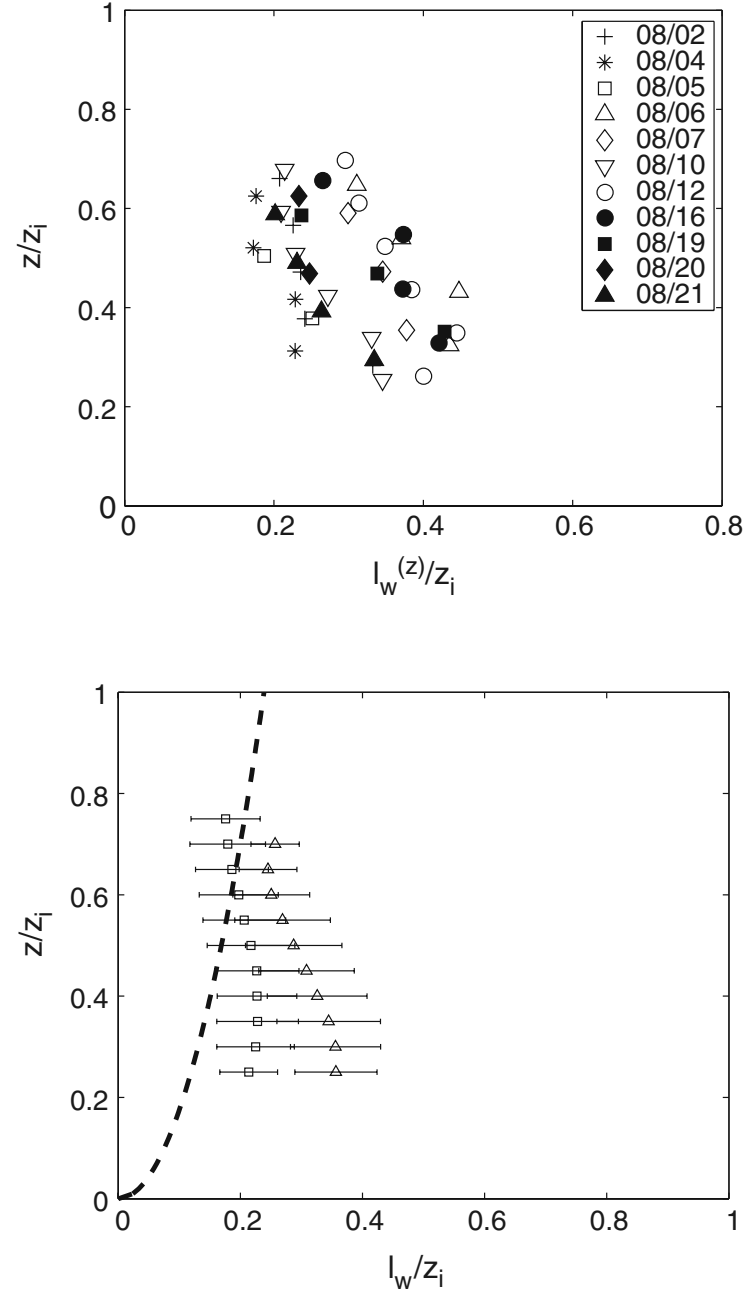

There seems to be no strong functional dependence of either $l_{w}^{(x)}(z)$ or $l_{w}^{(z)}(z)$ on $z_{i} / L_{o}$, although we note that the case with the smallest value of $z_{i} / L_{o}$ (5 August) is also at the small end of the observed values of $l_{w}^{(x)}(z) / z_{i}$ and similarly the case with the largest $z_{i} / L_{o}$ (16 August) is at the high end of the observed values of $l_{w}^{(x)}(z) / z_{i}$. This is consistent with more neutrally stable cases having somewhat smaller normalized $w$ eddy sizes, but the scatter in the data does not give a definitive functional dependency. There is even less evidence for any $z_{i} / L_{o}$ dependency in the $l_{w}^{(z)}(z) / z_{i}$ data.

Figure 8 shows the averages of both integral scales over 11 days, which makes more obvious the constancy of $l_{w}^{(x)}(z) / z_{i}$ and the decrease of $l_{w}^{(z)}(z) / z_{i}$ with height through the upper part of the CBL, and thus the varying anisotropy of $w$ with height. In effect, the vertical eddies become even more squashed and anisotropic near the top of the CBL due to the capping inversion. We also note that at the lowest level (i.e., $\left.z / z_{i} \simeq 0.25\right), l_{w}^{(z)}(z) / z_{i}$ increases with height, which is what one would expect near the surface. 

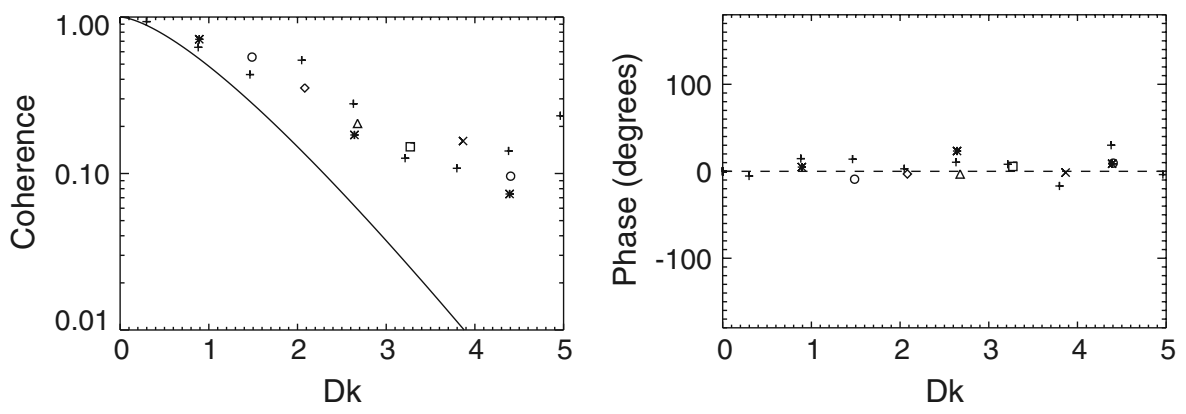

Fig. 9 Coherence (top panel) and phase (bottom panel) on 2 August 1996, between two levels se-

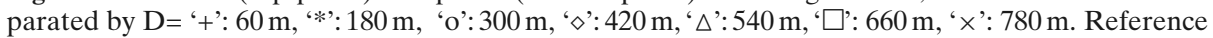
level is $0.25 z_{i}$. The solid line is the Kolmogorov model
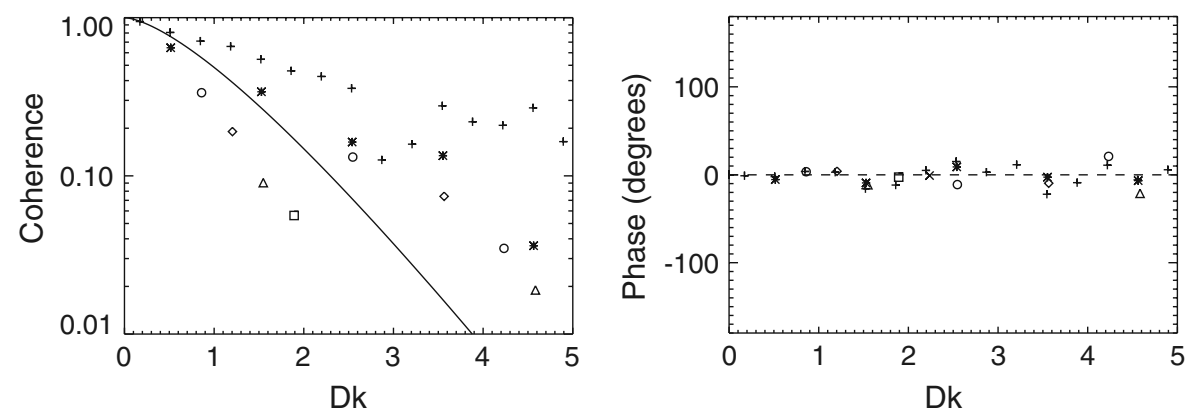

Fig. 10 Same as Fig. 9 for 4 August 1996

\section{Observed coherence}

The coherence $\operatorname{Coh}_{22}(D k)$ and phase $\phi_{22}(D k)$ were calculated for all the LIFT cases using $d f=50$ for all the periods indicated in Table 1 for $0.25<z / z_{i}<0.8$ in increments of $120 \mathrm{~m}$, and with $0.25 z_{i}$ as the reference level. This level is high enough to ensure that the calculations are carried out above the surface layer. From Kristensen and Kirkegaard (1986), the bias in the coherence for $d f=50$ is about 0.02 when the true coherence is zero.

\subsection{Departure from Kolmogorov model}

Figures 9 and 10 show examples of $C_{0 h}(D k)$ and $\phi_{22}(D k)$ for 2 and 4 August 1996, respectively; the Kolmogorov model is shown for comparison. Although both cases have similar integral scales of about $200 \mathrm{~m}$, the coherences differ between them. On 2 August, the observed coherence is always larger than the Kolmogorov model and does not seem to depend on $D / L$, but on 4 August, for small values of $D k$ but large values of $D$, the observed coherence falls below the Kolmogorov model; i.e., the coherence is also a function of $D / L$. As pointed out previously for the von Kármán spectrum, the coherence decreases with increasing $D / L$, especially at low wavenumbers, where the predicted coherence does not go to one for $D k \rightarrow 0$. 
Fig. 11 Observed coherence for all 11 LIFT days with $D / L=0.15 \pm 0.05$. The dashed line is the Kolmogorov model, the solid line the von Kármán model

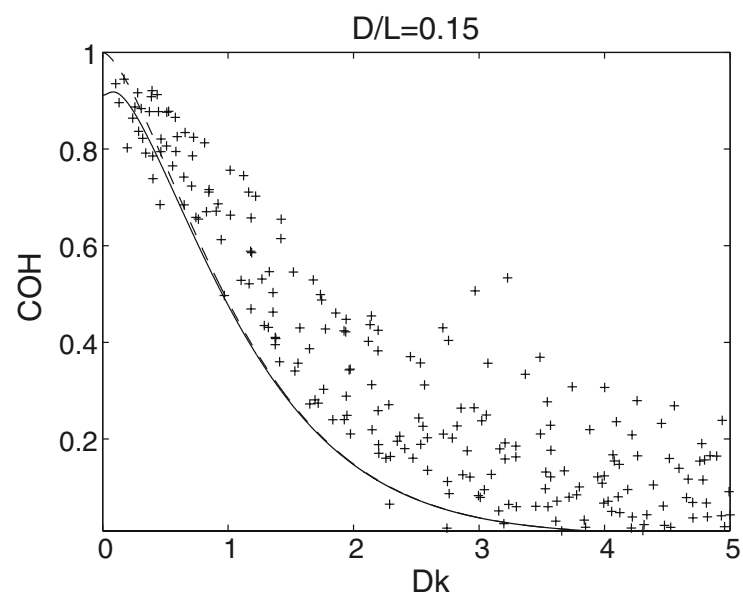

Figures 9 and 10 show that the phase angle is insignificantly different from zero, indicating that there is negligible tilt in $w$ with $z$ over the depth of CBL probed. This was true for all of the LIFT cases; the average phase shift was always $<3^{\circ}$, which amounts to a tilt of $<5 \mathrm{~m}$ for a height increment of $100 \mathrm{~m}$. The same observation was made by Mayor and Lenschow (2002) based on two LIFT cases.

\subsection{Departure from von Kármán model}

Figures 11 and 12 display the coherence for all 11 LIFT days as a function of $D k$ for two particular values of $D / L$, where $L$ was obtained from $l_{w}^{(x)}(z)$ via (10). All the points for which $D / L=0.55 \pm 0.10$ are plotted in Fig. 11 and all the points for which $D / L=0.15 \pm 0.10$ are plotted in Fig. 12. Both Kolmogorov and von Kármán predictions are also displayed. In the first case, $D / L$ is small enough for the two theories to be negligibly different for $D k>0.5$. The agreement with the von Kármán model is generally good for small $D k$, but the observed coherence is consistently larger than the predicted for $D k>0.5$. For the second case (Fig. 12) the scatter is larger, likely because departures from assumed isotropic turbulence become increasingly likely as $D / L$ increases, and these departures seem to be different for different cases, so it is difficult to discern any consistency in the departures. But the cloud of points does show a smaller coherence for small $D k$ than for the first case as predicted by the von Kármán model.

Another way to check the effect of the integral scale on the coherence is to keep the ratio of the two variables $D / L$ and $D k$ constant - that is keep $k L$ constant. The curves obtained for the von Kármán coherence as a function of $D k$ lie increasingly below the Kolmogorov coherence with an increasing slope as $k L$ decreases. Figures 13 and 14 show the measured and modelled coherence for two separate days each with different ranges of values for $k L$. We can see that the 5 August data points (Fig. 13) for $k L=0.7$ and 2.1 follow the predicted von Kármán model curves for the first few values of $D k$, then fall below the predicted curve, while for $k L=3.5$, the points lie above the predicted curve for small $D k$ but lie close to the predicted curve for large $D k$. For $k L>3.5$, the points lie above the predicted curve for all values of $D k$. For this particular day, the mean wind speed was high $\left(8.6 \mathrm{~m} \mathrm{~s}^{-1}\right)$ while $l_{w}^{(x)}$ was small; that 
Fig. 12 Same as Fig. 11 for $D / L=0.55 \pm 0.05$

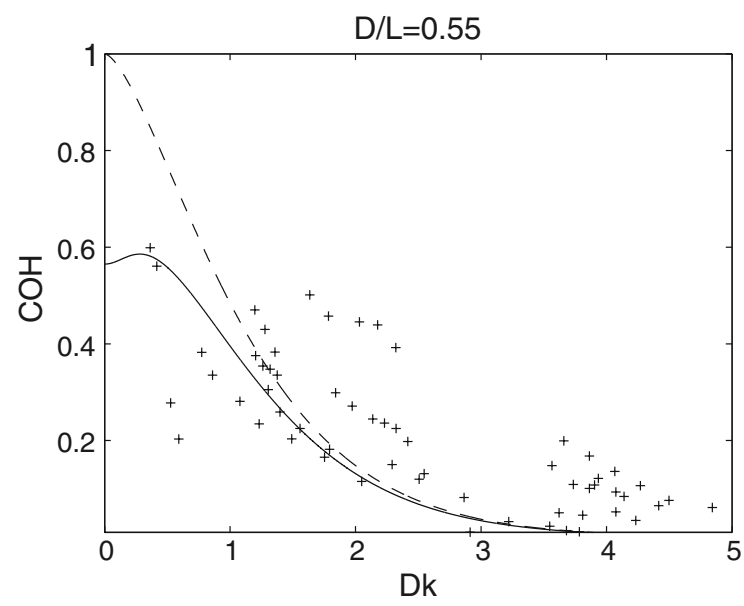

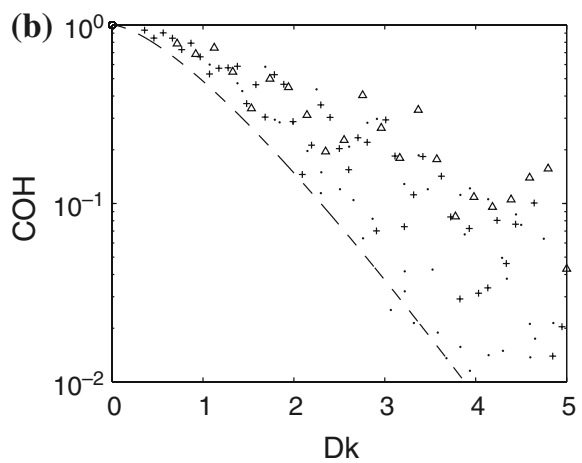

Fig. 13 Coherence observed on 5 August 1996 for different values of $k$ : (a)‘o' $-k=0.0017 \mathrm{~m}^{-1}(k L=$ $0.7) ;{ }^{\prime}+{ }^{\prime}-k=0.0051 \mathrm{~m}^{-1}(k L=2.1) ; ' \triangle^{\prime}-k=0.0085 \mathrm{~m}^{-1}(k L=3.5)$. The von Kármán model predictions corresponding to these wave numbers are given by the thick solid line, the thick dashed line, and the thin solid line, respectively, while the thin dashed line is the Kolmogorov model prediction. (b) $k>0.0085 \mathrm{~m}^{-1}$ with different values of $D$ : 'o' $-D=0 \mathrm{~m}$; ' $\triangle$ ' $-D=30 \mathrm{~m}$, '+' $-D=60 \mathrm{~m}$; and '.' $-D>60 \mathrm{~m}$. The dashed line is the prediction for both Kolmogorov and von Kármán models

is, $k L$ is small enough that the smallest increment in $k$ reveals a difference between the two theories.

For 16 August(Fig. 14), the Kolmogorov and the von Kármán models give almost identical results. The wind is light $\left(2.2 \mathrm{~m} \mathrm{~s}^{-1}\right)$ so that $k L$ is large. For values of $k L \geq 7.2$ all the points exceed the modelled curve.

These results show a consistently larger observed coherence than predicted by the von Kármán model when $k L>3$. This suggests that either the von Kármán spectral shape does not adequately represent the actual spectra or that the turbulence becomes increasingly anisotropic as $k L$ becomes larger.

To investigate whether horizontal convective rolls play a role in this observed anisotropy, we used the criteria for rolls given by Weckwerth (1999) to distinguish low-probability roll days (due to large values of $-z_{i} / L_{o}$ ) from days where rolls are more likely. Comparison of the autocorrelation functions and spectra on the 

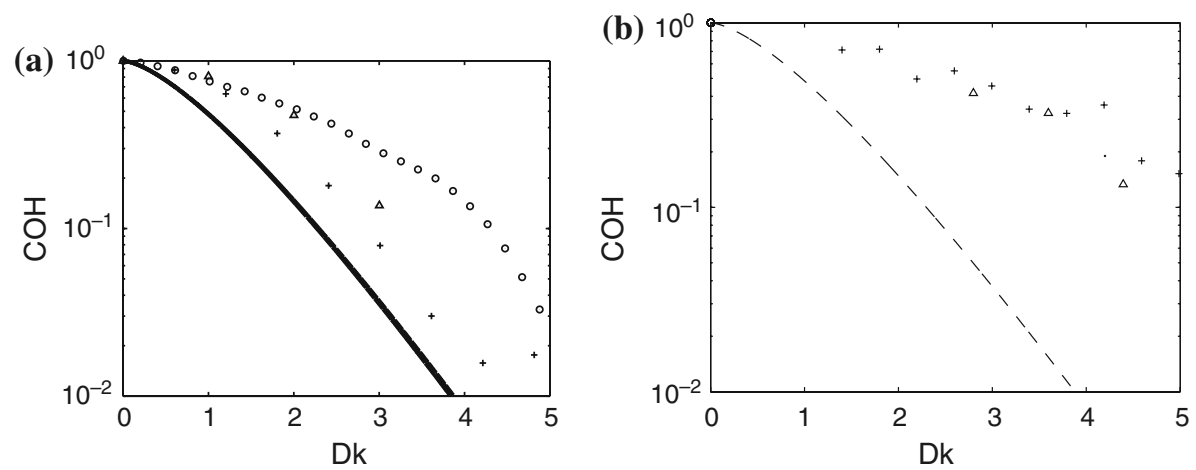

Fig. 14 Coherence observed on 16 August 1996 for different values of $k$ : (a) 'o' $-k=$ $0.0068 \mathrm{~m}^{-1}(k L=7.2)$; ' ${ }^{\prime}-k=0.0201 \mathrm{~m}^{-1}(k L=21.4) ;{ }^{\circ} \triangle^{\prime}-k=0.0334 \mathrm{~m}^{-1}(k L=35.6)$. The von Kármán model predictions corresponding to these wave numbers, defined as in Fig. 13, overlap the Kolmogorov prediction. (b) $k>0.0334 \mathrm{~m}^{-1}$ with different values of $D$ : 'o' $-D=0 \mathrm{~m}$; ' $\triangle$ ' $-D=30 \mathrm{~m}$; ' + ' $-D=60 \mathrm{~m}$; and ${ }^{\prime}$ ' $-D>60 \mathrm{~m}$. The dashed line is the prediction for both Kolmogorov and von Kármán models

low-probability roll days of 2, 10, 16, and 21 August 1996 with the remaining days, which were high-probability roll days, showed no obvious systematic differences.

\section{Summary}

Measurements from a ground-based zenith-pointing Doppler lidar collected during LIFT enabled us to obtain two-dimensional fields of $w$ for extended mid-day periods in the CBL above $\simeq 390 \mathrm{~m}$. These measurements have been used to calculate, for the first time, integral scales of $w$ in both the vertical and alongwind directions, and coherence of $w$ between two levels. The alongwind integral scale is approximately constant with height, in contrast to in situ aircraft results from AMTEX (in a convective marine boundary layer) that showed an increase with height. We speculate that this might be a result of stratiform cloud in AMTEX or the surface heterogeneity in LIFT. The vertical integral scale decreases with height. We know of no other observations with which this can be compared. We found that the vertical and horizontal integral scales correlate well with each other, and that the ratio of the vertical scale to the horizontal scale is $\simeq 1.3$ in the middle of the CBL, that is, 0.65 times what would be the case for isotropic turbulence. This ratio decreases with height through the upper $2 / 3$ of the CBL.

We observed larger coherence of the vertical velocity along the vertical than predicted by isotropic turbulence, especially as $D k$ and $D / L$ become large. Thus, not surprisingly, the larger the separation and the larger the wavenumber, the more anisotropic the turbulence. We also found no significant tilt of the thermal structures throughout our measured domain, which is roughly $>0.2 z_{i}$; that is, wind shear is too small to affect the orientation of thermals for $z>0.2 z_{i}$.

Acknowledgements The LIFT experiment was funded by the NCAR Atmospheric Technology Division Director's Office and the Department of Energy/OAGR. This work was made possible thanks to the MMM and ATD divisions of NCAR. The authors would like to thank Wayne Angevine, 
Rod Frehlich, Michael Hardesty, Leif Kristensen, and the two anonymous reviewers for their useful comments.

\section{References}

Angevine WM, Grimsdell AW, Hartten LM, Delany AC (1998) The flatland boundary layer experiments. Bull Amer Meteorol Soc 79:419-431

Batchelor GK (1953) Homogeneous turbulence Cambridge University Press, UK, 197 pp.

Cohn SA, Angevine WM (2000) Boundary layer height and entrainment zone thickness measured by lidars and wind-profiling radars. J Appl Meteorol 39:1233-1247

Cohn SA, Mayor SD, Grund TM, Weckwerth TM, Sneff C (1998) The lidars in flat terrain experiment LIFT. Bull Amer Meteorol Soc 79:1329-1343

Davis KJ (1992) surface fluxes of trace gases derived from convective-layer profiles. University of Colorado, Boulder, $\mathrm{CO}$

Durand P, Thoumieux F, Lambert D (2000) Turbulent length-scales in the marine atmospheric mixed layer. Quart J Roy Meteorol Soc 126:1889-1912

Frehlich R (1997) Effects of wind turbulence on coherent Doppler Lidar performance. J Atmos Oceanic Tech 14:54-75

Frehlich R, Meillier Y, Jensen ML, Balsley B (2006) Measurements of boundary layer profiles in an urban environment. J Appl Meteorol in press

Grimsdell AW, Angevine WM (1998) Convective boundary layer height measurement with wind profilers and comparison to cloud base. J Atmos Oceanic Tech 15:1331-1338

Grimsdell AW, Angevine WM (2002) Observations of the afternoon transition of the convective boundary layer. J Appl Meteorol 41: 3-11

Grund CJ, Banta RM, George JL, Howell JN, Post MJ, Richter RA, Weickman AM (2001) High-resolution doppler lidar for boundary layer and cloud research. J Atmos Oceanic Tech 18:376

Kristensen L, Jensen NO (1979) Lateral coherence in isotropic turbulence and in the natural wind. Boundary-Layer Meteorol 17:353-373

Kristensen L, Kirkegaard P (1986) Sampling problems with spectral coherence, Technical report Riso-R-526. Riso National Laboratory, DK-4000, Roskilde, Denmark

Kristensen L, Lenschow DH, Kirkegaard P, Courtney M (1989) The spectral velocity tensor for homogeneous boundary layer turbulence. Boundary-Layer Meteorol 47:149-193

Lenschow DH, Kristensen L (1988) Applications of dual aircraft formation flights. J Atmos Oceanic Tech 5:715-726

Lenschow DH, Krummel PB, Siems ST (1999) Measuring entrainment, divergence and vorticity on the mesoscale from aircraft. J Atmos Oceanic Tech 16:1384-1400

Lenschow DH, Stankov BB (1986) Length scales in the convective boundary layer. J Atmos Sci 43:1198-1209

Lenschow DH, Wulfmeyer V, Senff C (2000) Measuring second- through fourth-order moments in noisy data. J Atmos Oceanic Tech 17:1330-1347

Luke YL (1972) Integral of Bessel functions, Handbook of Mathematical Functions Abramowitz M. In: Stegun I. (eds) Dover publications Inc. New York, pp 479-494

Mann J (1994) The spatial structure of neutral atmospheric surface-layer turbulence. J Fluid Mech 273:141-168

Mayor S, Lenschow DH (2002) Coherence of vertical velocity from a zenith-pointing Doppler lidar. In: 15th Symposium on boundary layers and turbulence, Wageningen, The Netherlands, 15-19 July 2002

Prueger JH, Kustas WP, Hipps LE, Hatfield JL, Cahill A, Williams CA, Albertson JD, Eichinger WE, Cooper DI, Brunsell N, Gillies R (2003) Spatial variability of turbulent fluxes across a corn/soybean production region in central Iowa'. AMS 17th Conference on Hydrology, Long Beach, CA, USA

Weckwerth T (1999) An observational study of the evolution of horizontal convective rolls. Mon Wea Rev 127:2160-2179 